\title{
ON FOURNIER-GAGLIARDO MIXED NORM SPACES
}

\author{
Robert Algervik and Viktor I. Kolyada \\ Karlstad University, Department of Mathematics \\ Universitetsgatan 1, 65188 Karlstad, Sweden; robealge@kau.se \\ Karlstad University, Department of Mathematics \\ Universitetsgatan 1, 65188 Karlstad, Sweden; viktor.kolyada@kau.se
}

\begin{abstract}
We study mixed norm spaces $\mathscr{V}\left(\mathbf{R}^{n}\right)$ that arise in connection with embeddings of Sobolev spaces $W_{1}^{1}\left(\mathbf{R}^{n}\right)$. We prove embeddings of $\mathscr{V}\left(\mathbf{R}^{n}\right)$ into Lorentz type spaces defined in terms of iterative rearrangements. Basing on these results, we introduce the scale of mixed norm spaces $\mathscr{V}^{p}\left(\mathbf{R}^{n}\right)$. We prove that $\mathscr{V} \subset \mathscr{V}^{p}$ and we discuss some questions related to this embedding.
\end{abstract}

\section{Introduction}

Let $W_{p}^{1}\left(\mathbf{R}^{n}\right)(1 \leq p<\infty)$ be the Sobolev space of all functions $f \in L^{p}\left(\mathbf{R}^{n}\right)$ for which all first-order weak derivatives $\partial f / \partial x_{k} \equiv D_{k} f$ exist and belong to $L^{p}\left(\mathbf{R}^{n}\right)$. The classical Sobolev theorem asserts that for any function $f$ in $W_{p}^{1}\left(\mathbf{R}^{n}\right)(1 \leq p<n)$

$$
\|f\|_{q^{*}} \leq c \sum_{k=1}^{n}\left\|D_{k} f\right\|_{p}, \quad q^{*}=\frac{n p}{n-p} .
$$

Sobolev proved this inequality in 1938 for $p>1$. For $p=1$ inequality (1.1) was proved independently by Gagliardo (1958) and Nirenberg (1959). The core of Gagliardo's approach [7] is the following:

Lemma 1.1. Let $n \geq 2$. Assume that $g_{k} \in L^{1}\left(\mathbf{R}^{n-1}\right)(k=1, \ldots, n)$ are nonnegative functions on $\mathbf{R}^{n-1}$. Then

$$
\int_{\mathbf{R}^{n}} \prod_{k=1}^{n} g_{k}\left(\hat{x}_{k}\right)^{1 /(n-1)} d x \leq\left(\prod_{k=1}^{n} \int_{\mathbf{R}^{n-1}} g_{k}\left(\hat{x}_{k}\right) d \hat{x}_{k}\right)^{1 /(n-1)} .
$$

As usual, for any vector $x \in \mathbf{R}^{n}$ and any $k=1, \ldots, n$ we denote by $\hat{x}_{k}$ the $(n-1)$-dimensional vector obtained from $x$ by removal of its $k$ th coordinate. We write $x=\left(x_{k}, \hat{x}_{k}\right)$.

Assume now that $f \in W_{1}^{1}\left(\mathbf{R}^{n}\right)$. Then for any $k=1, \ldots, n$ and almost all $x \in \mathbf{R}^{n}$,

$$
f(x)=\int_{-\infty}^{x_{k}} D_{k} f\left(u, \hat{x}_{k}\right) d u=-\int_{x_{k}}^{\infty} D_{k} f\left(u, \hat{x}_{k}\right) d u .
$$

It follows that for almost all $x \in \mathbf{R}^{n}$

$$
|f(x)| \leq \frac{1}{2} \int_{\mathbf{R}}\left|D_{k} f\left(u, \hat{x}_{k}\right)\right| d u \equiv g_{k}\left(\hat{x}_{k}\right), \quad k=1, \ldots, n .
$$


Thus,

$$
|f(x)| \leq \frac{1}{2}\left(\prod_{k=1}^{n} g_{k}\left(\hat{x}_{k}\right)\right)^{1 / n} .
$$

Using this estimate and applying Lemma 1.1, we obtain

$$
\|f\|_{n /(n-1)} \leq \frac{1}{2}\left(\prod_{k=1}^{n}\left\|D_{k} f\right\|_{1}\right)^{1 / n} .
$$

This implies inequality (1.1) for $p=1$. However, a more general statement can be derived from (1.2). Let

$$
\mathscr{V}_{k}\left(\mathbf{R}^{n}\right) \equiv L_{\hat{x}_{k}}^{1}\left(\mathbf{R}^{n-1}\right)\left[L_{x_{k}}^{\infty}(\mathbf{R})\right], \quad 1 \leq k \leq n,
$$

be the space of measurable functions on $\mathbf{R}^{n}$ with the finite mixed norm

$$
\|f\|_{\mathscr{V}_{k}} \equiv\left\|\psi_{k}\right\|_{L^{1}\left(\mathbf{R}^{n-1}\right)}, \quad \text { where } \psi_{k}\left(\hat{x}_{k}\right)=\operatorname{ess}_{\sup _{x_{k} \in \mathbf{R}}}|f(x)| \text {. }
$$

We observe that $\|f\|_{\mathscr{V}_{k}}$ has a clear geometric interpretation: it is the $n$-dimensional measure of the essential projection of the set

$$
\left\{(x, y) \in \mathbf{R}^{n} \times[0 ; \infty): 0 \leq y \leq|f(x)|\right\}
$$

into the hyperplane $x_{k}=0$ (see Theorem 3.1 below).

Throughout this paper, we denote also

$$
\mathscr{V}\left(\mathbf{R}^{n}\right)=\bigcap_{k=1}^{n} \mathscr{V}_{k}\left(\mathbf{R}^{n}\right), \quad\|f\|_{\mathscr{V}}=\sum_{k=1}^{n}\|f\|_{\mathscr{V}_{k}} .
$$

Note that by (1.3), for any function $f \in W_{1}^{1}\left(\mathbf{R}^{n}\right)$

$$
\|f\|_{\mathscr{V}_{k}} \leq \frac{1}{2}\left\|D_{k} f\right\|_{1} \quad(1 \leq k \leq n)
$$

Gagliardo's lemma immediately implies the following theorem:

Theorem 1.2. Let $n \geq 2$. If $f \in \mathscr{V}\left(\mathbf{R}^{n}\right)$, then $f \in L^{n^{\prime}}\left(\mathbf{R}^{n}\right)$ and

$$
\|f\|_{n^{\prime}} \leq\left(\prod_{k=1}^{n}\|f\|_{\mathscr{V}_{k}}\right)^{1 / n}
$$

As usual, for any $1<p<\infty$ we denote $p^{\prime}=p /(p-1)$. By (1.7), Theorem 1.2 implies (1.4).

It is well known that the left-hand side in (1.1) can be replaced by a stronger Lorentz norm $\|f\|_{q^{*}, p}$. Recall that the Lorentz space $L^{q, p}\left(\mathbf{R}^{n}\right)(0<q, p<\infty)$ is defined as the class of all measurable functions $f$ on $\mathbf{R}^{n}$ such that

$$
\|f\|_{q, p} \equiv\left(\int_{0}^{\infty}\left[t^{1 / q} f^{*}(t)\right]^{p} \frac{d t}{t}\right)^{1 / p}<\infty
$$

where $f^{*}$ denotes the nonincreasing rearrangement of $f$. Note that the quasi-norm $\|\cdot\|_{q, p}$ is a norm if and only if $1 \leq p \leq q<\infty$ (see [12]). For a fixed $q$, the Lorentz spaces $L^{q, p}$ increase as the secondary index $p$ increases (see [2, p. 217]).

The following strengthening of (1.1) holds:

$$
\|f\|_{q^{*}, p} \leq c \sum_{k=1}^{n}\left\|D_{k} f\right\|_{p}, \quad 1 \leq p<n, q^{*}=\frac{n p}{n-p}
$$


(see [15], [16] for $p>1$, [17] for $p=1$ ). In this paper we consider (1.8) only in the case $p=1$. There are numerous proofs of (1.8) in this limiting case; most of them are related to rearrangements, properties of level sets, and geometric inequalities. A very interesting approach given by Fournier [6] was based on the following refinement of Theorem 1.2.

Theorem 1.3. Let $n \geq 2$. If $f \in \mathscr{V}\left(\mathbf{R}^{n}\right)$, then $f \in L^{n^{\prime}, 1}\left(\mathbf{R}^{n}\right)$ and

$$
\|f\|_{n^{\prime}, 1} \leq n^{\prime}\left(\prod_{k=1}^{n}\|f\|_{\mathscr{V}_{k}}\right)^{1 / n} .
$$

By virtue of (1.7), inequality (1.9) immediately implies (1.8) for $p=1$. Thus, embedding

$$
W_{1}^{1}\left(\mathbf{R}^{n}\right) \subset L^{n^{\prime}, 1}\left(\mathbf{R}^{n}\right)
$$

can be split into two successive steps

$$
W_{1}^{1}\left(\mathbf{R}^{n}\right) \subset \mathscr{V}\left(\mathbf{R}^{n}\right) \quad \text { and } \quad \mathscr{V}\left(\mathbf{R}^{n}\right) \subset L^{n^{\prime}, 1}\left(\mathbf{R}^{n}\right) .
$$

Note that similar splitting for embedding $W_{p}^{1}\left(\mathbf{R}^{n}\right) \subset L^{q^{*}, p}\left(\mathbf{R}^{n}\right)$ in the whole range $1 \leq p<n$ was obtained in [9]. Different extensions of Theorem 1.3 and their applications have been studied in the works [3], [9], [14].

The motivation for this paper was twofold. On the one hand, it was motivated by Theorems 1.2 and 1.3. These theorems show that the integrability properties of functions of several variables can be controlled by the behaviour of the $L^{\infty}$-norms of their linear sections. Following this idea, we obtain stronger versions of inequality (1.9) expressed in terms of iterative rearrangements (see Sections 2 and 4 below). We observe that these results were also inspired by embeddings of Sobolev spaces into modified Lorentz spaces proved in [8].

On the other hand, smoothness or integrability properties of functions reflect on the behaviour of their linear sections. In particular, inequality (1.7) shows that for any $f \in W_{1}^{1}\left(\mathbf{R}^{n}\right)$ the $L^{\infty}$-norms of $x_{k}$-sections $f_{\hat{x}_{k}}\left(x_{k}\right)=f\left(x_{k}, \hat{x}_{k}\right)$ are integrable functions of $\hat{x}_{k}$ in $\mathbf{R}^{n-1}$. It is also natural to study other norms of linear sections. For example, let us consider $L^{1}$-norms. Assume that $f \in W_{1}^{1}\left(\mathbf{R}^{n}\right)$ and set

$$
\varphi_{k}\left(\hat{x}_{k}\right)=\int_{\mathbf{R}}|f(x)| d x_{k} \quad(k=1, \ldots, n) .
$$

If $n=2$, then $\varphi_{k} \in L^{\infty}(\mathbf{R})(k=1,2)$ (it follows from (1.7)). Let $n \geq 3$. It is easily seen that $\varphi_{k} \in W_{1}^{1}\left(\mathbf{R}^{n-1}\right)(k=1, \ldots, n)$. Applying inequality (1.8), we obtain that $\varphi_{k} \in L^{(n-1)^{\prime}, 1}\left(\mathbf{R}^{n-1)}\right)$. Thus,

$$
W_{1}^{1}\left(\mathbf{R}^{n}\right) \subset \bigcap_{k=1}^{n} L_{\hat{x}_{k}}^{(n-1)^{\prime}, 1}\left(\mathbf{R}^{n-1}\right)\left[L_{x_{k}}^{1}(\mathbf{R})\right] .
$$

These observations (together with embeddings proved in Section 4) led us to the definition of the scale of mixed norm spaces

$$
\mathscr{V}^{p}\left(\mathbf{R}^{n}\right)=\bigcap_{k=1}^{n} L_{\hat{x}_{k}}^{p, 1}\left(\mathbf{R}^{n-1}\right)\left[L_{x_{k}}^{r_{p}, 1}(\mathbf{R})\right]
$$


$\left(1 \leq p \leq(n-1)^{\prime}, r_{p}=p^{\prime} /(n-1)\right)$. For $p=1$ we have $\mathscr{V}^{1}\left(\mathbf{R}^{n}\right)=\mathscr{V}\left(\mathbf{R}^{n}\right)$ (see Section 5) and thus the space $\mathscr{V}$ is included to the scale. We prove that

$$
\mathscr{V}\left(\mathbf{R}^{n}\right) \subset \mathscr{V}^{p}\left(\mathbf{R}^{n}\right), \quad 1 \leq p \leq(n-1)^{\prime}, n \geq 2 .
$$

By virtue of the first embedding in (1.11), for $n \geq 3$ and $p=(n-1)^{\prime}(1.14)$ implies (1.12). We obtain also some results concerning endpoints in the estimates of $\mathscr{V}^{p_{-}}$ norms (see Remark 5.4 and Theorem 5.7 below).

In this paper we do not study relations between spaces $\mathscr{V}^{p}$ with different values of $p$. We notice only that this problem is not immediate. In particular, the spaces $\mathscr{V}^{p}\left([0,1]^{2}\right)$ do not form a monotone scale.

We observe also that the scales of spaces of the type (1.13) provide a flexible control of the growth of linear sections of functions. As in the works [3], [6], [9], [14], embeddings of these spaces can be applied to obtain optimal results in various problems.

Acknowledgements. The authors are grateful to the referee for his/her useful remarks.

\section{Iterative rearrangements}

For a measurable set $E \subset \mathbf{R}^{k}$, we denote by $\operatorname{mes}_{k} E$ the Lebesgue measure of $E$ in $\mathbf{R}^{k}$. Let $f$ be a measurable function on $\mathbf{R}^{n}$. Recall that nonincreasing rearrangement of $f$ is a nonnegative and nonincreasing function $f^{*}$ on $\mathbf{R}_{+} \equiv(0,+\infty)$ which is equimeasurable with $|f|$ (see [2, p. 37]). We assume in addition that the rearrangement is left continuous on $\mathbf{R}_{+}$(then it is defined uniquely). By $S_{0}\left(\mathbf{R}^{n}\right)$ we denote the class of all measurable and almost everywhere finite functions $f$ on $\mathbf{R}^{n}$, for which

$$
\operatorname{mes}_{n}\left\{x \in \mathbf{R}^{n}:|f(x)|>y\right\}<\infty \text { for all } y>0 \text {. }
$$

It is easy to see that $f \in S_{0}\left(\mathbf{R}^{n}\right)$ if and only if $f^{*}(t) \rightarrow 0$ as $t \rightarrow+\infty$.

Further, we consider rearrangements with respect to specific variables. Let $f \in$ $S_{0}\left(\mathbf{R}^{n}\right)$ and let $1 \leq k \leq n$. Fix $\hat{x}_{k} \in \mathbf{R}^{n-1}$, and consider the function $f_{\hat{x}_{k}}\left(x_{k}\right)=$ $f\left(x_{k}, \hat{x}_{k}\right)$. By Fubini's theorem, $f_{\hat{x}_{k}} \in S_{0}(\mathbf{R})$ for almost all $\hat{x}_{k} \in \mathbf{R}^{n-1}$. We denote the rearrangement of $f$ with respect to $x_{k}$ by $\mathscr{R}_{k} f$. That is, we set

$$
\mathscr{R}_{k} f\left(t, \hat{x}_{k}\right)=\left(f_{\hat{x}_{k}}\right)^{*}(t), \quad t>0 .
$$

This function is defined almost everywhere on $\mathbf{R}_{+} \times \mathbf{R}^{n-1}$. Moreover, $\mathscr{R}_{k} f$ is a measurable function equimeasurable with $f$ (see [8]). Let $\mathscr{P}_{n}$ denote the set of all permutations $\sigma=\left(k_{1}, \ldots, k_{n}\right)$ of the numbers $1,2, \ldots, n$. Let $f \in S_{0}\left(\mathbf{R}^{n}\right)$ and $\sigma \in \mathscr{P}_{n}$. The $\mathscr{R}_{\sigma}$-rearrangement of $f$ is defined as the function

$$
\mathscr{R}_{\sigma} f(t)=\mathscr{R}_{k_{n}} \cdots \mathscr{R}_{k_{1}} f(t), \quad t \in \mathbf{R}_{+}^{n} .
$$

That is, we obtain $\mathscr{R}_{\sigma} f$ from $f$ by "rearranging" $f$ succesively with respect to the variables $x_{k_{1}}, \ldots, x_{k_{n}}$, starting with $x_{k_{1}}$. In so doing, we replace successively the arguments $x_{k_{1}}, \ldots, x_{k_{n}}$ by the arguments $t_{k_{1}}, \ldots, t_{k_{n}}$. It is easy to see that $\mathscr{R}_{\sigma} f$ decreases monotonically with respect to each variable. In view of the above observation, $\mathscr{R}_{\sigma} f$ is equimeasurable with $|f|$.

In what follows we set

$$
\pi(t)=\prod_{k=1}^{n} t_{k}, \quad t \in \mathbf{R}_{+}^{n} .
$$


For $0<p, s<\infty$ and $\sigma \in \mathscr{P}_{n}$, the space $\mathscr{L}_{\sigma}^{p, s}\left(\mathbf{R}^{n}\right)$ is defined as the class of all functions $f \in S_{0}\left(\mathbf{R}^{n}\right)$ such that

$$
\|f\|_{\mathscr{L}_{\sigma}^{p, s}} \equiv\left(\int_{\mathbf{R}_{+}^{n}}\left[\pi(t)^{1 / p} \mathscr{R}_{\sigma} f(t)\right]^{s} \frac{d t}{\pi(t)}\right)^{1 / s}<\infty
$$

(see [4]). Set also

$$
\mathscr{L}^{p, s}\left(\mathbf{R}^{n}\right)=\bigcap_{\sigma \in \mathscr{P}_{n}} \mathscr{L}_{\sigma}^{p, s}\left(\mathbf{R}^{n}\right)
$$

It was proved in [18] that

$$
\|f\|_{p, s} \leq 2^{1 / s-1 / p}\|f\|_{\mathscr{L}_{\sigma}^{p, s}}
$$

for $0<s \leq p<\infty$ and $\sigma \in \mathscr{P}_{n}$. Thus, for any $\sigma \in \mathscr{P}_{n}$

$$
\mathscr{L}_{\sigma}^{p, s} \subset L^{p, s} \quad \text { if } \quad 0<s \leq p<\infty
$$

(for $p<s$ the converse embedding holds). The key point of the proof is the following: if a function $F$ defined on $\mathbf{R}_{+}^{n}$ is nonnegative and nonincreasing with respect to each variable, then for any $t \in \mathbf{R}_{+}^{n}$

$$
\operatorname{mes}_{n}\left\{s \in \mathbf{R}_{+}^{n}: F(s) \geq F(t)\right\} \geq \pi(t) .
$$

Basing on this observation, we give an alternative proof of (2.1) with a better constant.

Theorem 2.1. Let $f \in S_{0}\left(\mathbf{R}^{n}\right)$ and $\sigma \in \mathscr{P}_{n}$. For all $0<s \leq p<\infty$,

$$
\|f\|_{L^{p, s}} \leq\|f\|_{\mathscr{L}_{\sigma}^{p, s}} .
$$

Proof. Set $F(t)=\mathscr{R}_{\sigma} f(t)$. We may suppose that

$$
\operatorname{mes}_{n}\left\{t \in \mathbf{R}_{+}^{n}: F(t)=y\right\}=0
$$

for all $y \geq 0$. Fix $a>1$ and set

$$
A_{\nu}=\left\{t \in \mathbf{R}_{+}^{n}: f^{*}\left(a^{-\nu+1}\right) \leq F(t)<f^{*}\left(a^{-\nu}\right)\right\}, \quad \nu \in \mathbf{Z} .
$$

Let $t \in A_{\nu}$. Then by (2.3) and (2.5),

$$
\begin{aligned}
\pi(t) & \leq \operatorname{mes}_{n}\left\{s \in \mathbf{R}_{+}^{n}: F(s) \geq f^{*}\left(a^{-\nu+1}\right)\right\} \\
& =\operatorname{mes}_{1}\left\{u>0: f^{*}(u) \geq f^{*}\left(a^{-\nu+1}\right)\right\}=a^{-\nu+1} .
\end{aligned}
$$

Thus, we have

$$
\begin{aligned}
\|f\|_{\mathscr{L}_{\sigma}^{p, s}}^{s} & =\sum_{\nu \in \mathbf{Z}} \int_{A_{\nu}} \pi(t)^{s / p-1} F(t)^{s} d t \geq \sum_{\nu \in \mathbf{Z}} a^{(s / p-1)(-\nu+1)} \int_{A_{\nu}} F(t)^{s} d t \\
& =a^{s / p-1} \sum_{\nu \in \mathbf{Z}} a^{-\nu(s / p-1)} \int_{a^{-\nu}}^{a^{-\nu+1}} f^{*}(u)^{s} d u \\
& \geq a^{s / p-1} \sum_{\nu \in \mathbf{Z}} \int_{a^{-\nu}}^{a^{-\nu+1}} u^{s / p-1} f^{*}(u)^{s} d u=a^{s / p-1}\|f\|_{L^{p, s} .}^{s}
\end{aligned}
$$

Since $a>1$ is arbitrary, this implies inequality (2.4). 
Remark 2.2. Observe that embedding (2.2) is strict (see [18]). Moreover, if

$$
E=\left\{(x, y): 0<y \leq \frac{1}{x(\ln (2 / x))^{p / s}}, \quad 0<x \leq 1\right\}
$$

and $0<s<p<\infty$, then $|E|<\infty$, but the characteristic function of the set $E$ does not belong to $\mathscr{L}_{\{1,2\}}^{p, s}\left(\mathbf{R}^{2}\right) \cup \mathscr{L}_{\{2,1\}}^{p, s}\left(\mathbf{R}^{2}\right)$ (see [10, p. 55]).

In what follows we set

$$
\left\|\mathscr{R}_{\sigma} f\right\|_{\mathscr{V}_{k}\left(\mathbf{R}_{+}^{n}\right)} \equiv \int_{\mathbf{R}_{+}^{n-1}}\left\|\mathscr{R}_{\sigma} f\left(\hat{t}_{k}, \cdot\right)\right\|_{\infty} d \hat{t}_{k} \quad\left(\sigma \in \mathscr{P}_{n}, k=1, \ldots, n\right) .
$$

Lemma 2.3. Let $f \in S_{0}\left(\mathbf{R}^{n}\right)$. Then

$$
\left\|\mathscr{R}_{\sigma} f\right\|_{\mathscr{V}_{k}\left(\mathbf{R}_{+}^{n}\right)} \leq\|f\|_{\mathscr{V}_{k}\left(\mathbf{R}^{n}\right)} \quad(k=1, \ldots, n)
$$

for any $\sigma \in \mathscr{P}_{n}$ and any $k=1, \ldots, n$.

Proof. Let $\sigma \in \mathscr{P}_{n}$. We have

$$
|f(x)| \leq\left\|f\left(\hat{x}_{k}, \cdot\right)\right\|_{\infty} \equiv \psi_{k}\left(\hat{x}_{k}\right) \quad(k=1, \ldots, n)
$$

for almost all $x \in \mathbf{R}^{n}$. Hence, $\mathscr{R}_{\sigma} f(t) \leq \mathscr{R}_{\sigma_{k}} \psi_{k}\left(\hat{t}_{k}\right)$, where $\sigma_{k}$ is obtained from $\sigma$ by removing $k$. This gives

$$
\left\|\mathscr{R}_{\sigma} f\left(\hat{t}_{k}, \cdot\right)\right\|_{\infty} \leq \mathscr{R}_{\sigma_{k}} \psi_{k}\left(\hat{t}_{k}\right), \quad \hat{t}_{k} \in \mathbf{R}_{+}^{n-1} .
$$

Integrating this inequality over $\mathbf{R}_{+}^{n-1}$, and taking into account that

$$
\int_{\mathbf{R}_{+}^{n-1}} \mathscr{R}_{\sigma_{k}} \psi_{k}\left(\hat{t}_{k}\right) d \hat{t}_{k}=\int_{\mathbf{R}^{n-1}} \psi_{k}\left(\hat{x}_{k}\right) d \hat{x}_{k}=\|f\|_{\mathscr{V}_{k}\left(\mathbf{R}^{n}\right)}
$$

we obtain (2.6).

\section{Projections and spaces $\mathscr{V}_{k}$}

Let $E \subset \mathbf{R}^{n}$ be a measurable set and let $1 \leq k \leq n$. For a point $\hat{x}_{k} \in \mathbf{R}^{n-1}$, denote by $E\left(\hat{x}_{k}\right)$ the $\hat{x}_{k}$-section of the set $E$,

$$
E\left(\hat{x}_{k}\right)=\left\{x_{k} \in \mathbf{R}:\left(x_{k}, \hat{x}_{k}\right) \in E\right\} .
$$

By Fubini's theorem, for any $1 \leq k \leq n$ and almost all $\hat{x}_{k} \in \mathbf{R}^{n-1}$, the sections $E\left(\hat{x}_{k}\right)$ are measurable in $\mathbf{R}$, and the functions

$$
m_{k}\left(\hat{x}_{k}\right)=\operatorname{mes}_{1} E\left(\hat{x}_{k}\right), \quad k=1, \ldots, n,
$$

defined a.e. on $\mathbf{R}^{n-1}$, are measurable. The essential projection of $E$ into the coordinate hyperplane $x_{k}=0$ is defined to be the set $\Pi_{k}(E)$ of all points $\hat{x}_{k} \in \mathbf{R}^{n-1}$ such that $E\left(\hat{x}_{k}\right)$ is measurable and $m_{k}\left(\hat{x}_{k}\right)>0$. Since the function $m_{k}$ is measurable, the essential projection $\Pi_{k}(E)$ is measurable.

Let now $f$ be a measurable function on $\mathbf{R}^{n}$. Let $1 \leq k \leq n$. By Fubini's theorem, for almost all $\hat{x}_{k} \in \mathbf{R}^{n-1}$ the sections $f_{\hat{x}_{k}}$ are measurable functions on $\mathbf{R}$. Moreover, the function

$$
\psi_{k}\left(\hat{x}_{k}\right)=\left\|f_{\hat{x}_{k}}\right\|_{L^{\infty}(\mathbf{R})}=\operatorname{ess} \sup _{x_{k} \in \mathbf{R}}\left|f\left(x_{k}, \hat{x}_{k}\right)\right|
$$

(defined a.e. on $\mathbf{R}^{n-1}$ ) is measurable. It suffices to prove the latter statement in the case when $f$ is a bounded function with the compact support. In this case we have

$$
\psi_{k}\left(\hat{x}_{k}\right)=\lim _{\nu \rightarrow \infty}\left\|f_{\hat{x}_{k}}\right\|_{L^{\nu}(\mathbf{R})},
$$


and the functions $\hat{x}_{k} \mapsto\left\|f_{\hat{x}_{k}}\right\|_{L^{\nu}(\mathbf{R})}$ are measurable by Fubini's theorem.

Thus, the definition of the space $\mathscr{V}_{k}$ (see (1.5)) is correct. Now we shall show that the norm in $\mathscr{V}_{k}$ has a simple geometric interpretation. Let $f$ be a non-negative measurable function on $\mathbf{R}^{n}$ and let $U_{f}$ denote the region under the graph of $f$,

$$
U_{f}=\left\{(x, y) \in \mathbf{R}^{n} \times[0 ; \infty): 0 \leq y \leq f(x)\right\} .
$$

Theorem 3.1. Let $f$ be a nonnegative measurable function on $\mathbf{R}^{n}$ and let $\Pi_{k}\left(U_{f}\right)$ be the essential projection of $U_{f}$ into the hyperplane $x_{k}=0(1 \leq k \leq n)$. Then $f \in \mathscr{V}_{k}\left(\mathbf{R}^{n}\right)$ if and only if $\operatorname{mes}_{n} \Pi_{k}\left(U_{f}\right)<\infty$. Moreover, in this case

$$
\|f\|_{\mathscr{V}_{k}}=\operatorname{mes}_{n} \Pi_{k}\left(U_{f}\right) .
$$

Proof. We consider the case $k=n$ and set $A=\Pi_{n}\left(U_{f}\right)$. The set $A$ consists of all points $\left(\hat{x}_{n}, y\right)$ such that $\hat{x}_{n} \in \mathbf{R}^{n-1}, 0 \leq y<\infty$, the function $f_{\hat{x}_{n}}$ is measurable on $\mathbf{R}$, and

$$
\operatorname{mes}_{1}\left\{x_{n} \in \mathbf{R}: f\left(x_{n}, \hat{x}_{n}\right) \geq y\right\}>0 .
$$

Let a point $\hat{x}_{n} \in \mathbf{R}^{n-1}$ be such that $f_{\hat{x}_{n}}$ is measurable on $\mathbf{R}$. First, assume that $\psi_{n}\left(\hat{x}_{n}\right)<\infty$ (see (3.1)) and $\psi_{n}\left(\hat{x}_{n}\right)<y<\infty$. Then (3.2) does not hold and $\left(\hat{x}_{n}, y\right) \notin A$. Now, let $\psi_{n}\left(\hat{x}_{n}\right)>0$ and $0 \leq y<\psi_{n}\left(\hat{x}_{n}\right)$. Then, by the definition of essential supremum,

$$
\operatorname{mes}_{1}\left\{x_{n} \in \mathbf{R}: f\left(x_{n}, \hat{x}_{n}\right)>y\right\}>0
$$

and hence $\left(\hat{x}_{n}, y\right) \in A$. We obtain that

$$
\psi_{n}\left(\hat{x}_{n}\right)=\operatorname{mes}_{1}\left\{y>0:\left(\hat{x}_{n}, y\right) \in A\right\} .
$$

Thus,

$$
\int_{\mathbf{R}^{n-1}} \psi_{n}\left(\hat{x}_{n}\right) d \hat{x}_{n}=\operatorname{mes}_{n} A .
$$

On the other hand, by the definition, the latter integral is equal to $\|f\|_{\mathscr{V}_{n}}$. This proves the theorem.

Fournier [6, Theorem 3.1] proved the following theorem (see (1.6)).

Theorem 3.2. Let $f \in \mathscr{V}\left(\mathbf{R}^{n}\right)(n \geq 2)$ be a nonnegative function. Then $f \in$ $S_{0}\left(\mathbf{R}^{n}\right)$. Assume that a function $g$ defined on $\mathbf{R}^{n}$ is equimeasurable with $f$ and has the property that for each $y>0$ the set

$$
\left\{x \in \mathbf{R}^{n}: g(x)>y\right\}
$$

is essentially a cube in $\mathbf{R}^{n}$ with edges parallel to the coordinate axes. Then $\|g\|_{\mathscr{V}} \leq$ $\|f\|_{\mathscr{V}}$.

The proof of this theorem employed the following Loomis-Whitney isoperimetric inequality [11] (this inequality follows also from (1.2)).

Theorem 3.3. Let $E \subset \mathbf{R}^{n}$ be a measurable set. Then

$$
\left(\operatorname{mes}_{n} E\right)^{n-1} \leq \prod_{k=1}^{n} \operatorname{mes}_{n-1} \Pi_{k}(E) .
$$

We observe that Theorems 3.1 and 3.3 combined give a shorter proof of Theorem 3.2. 


\section{Iterative rearrangement inequalities}

As we observed in Section 1, Theorem 1.3 implies Sobolev-type inequality (1.8) for $p=1$. It was proved in [8] that the $L^{q^{*}, p}$-norm on the left-hand side in (1.8) can be replaced by the stronger $\mathscr{L}^{q^{*}, p}$-norm.

Theorem 4.1. Let $n \geq 2$ and $1 \leq p<n$. Set $q^{*}=n p /(n-p)$. If $f \in W_{p}^{1}\left(\mathbf{R}^{n}\right)$, then $f \in \mathscr{L}^{q^{*}, p}\left(\mathbf{R}^{n}\right)$ and

$$
\|f\|_{\mathscr{L} q^{*}, p} \leq c \sum_{k=1}^{n}\left\|D_{k} f\right\|_{p} .
$$

We obtain a similar refinement for mixed norm spaces $\mathscr{V}$. Denote by $\mathscr{M}_{\mathrm{dec}}\left(\mathbf{R}_{+}^{n}\right)$ the class of all nonnegative functions on $\mathbf{R}_{+}^{n}$ which are nonincreasing in each variable. For any $f \in \mathscr{M}_{\text {dec }}\left(\mathbf{R}_{+}^{n}\right)$ and any $\sigma \in \mathscr{P}_{n}$ we have that $f=\mathscr{R}_{\sigma} f$ a.e. on $\mathbf{R}_{+}^{n}$.

Our main result is the following:

Theorem 4.2. Let $n \geq 2,1 \leq p_{1}, \ldots, p_{n}<\infty$, and

$$
\sum_{k=1}^{n} \frac{1}{p_{k}^{\prime}}=1 \text {. }
$$

Assume that $f \in \mathscr{V}\left(\mathbf{R}^{n}\right)$. Then

$$
\int_{\mathbf{R}_{+}^{n}} \prod_{k=1}^{n} t_{k}^{1 / p_{k}-1} \mathscr{R}_{\sigma} f(t) d t \leq \prod_{k=1}^{n}\left(p_{k} p_{k}^{\prime}\|f\|_{\mathscr{V}_{k}}\right)^{1 / p_{k}^{\prime}}
$$

for any $\sigma \in \mathscr{P}_{n}$.

Proof. By virtue of Lemma 2.3, it is sufficient to prove inequality (4.3) for a function $f \in \mathscr{M}_{\mathrm{dec}}\left(\mathbf{R}_{+}^{n}\right)$. Set

$$
A_{j}=\left\{t \in \mathbf{R}_{+}^{n}: t_{j} \leq \prod_{k=1}^{n} t_{k}^{1 / p_{k}^{\prime}}\right\} \quad(j=1, \ldots, n) .
$$

Then

$$
\bigcup_{j=1}^{n} A_{j}=\mathbf{R}_{+}^{n}
$$

Indeed, set

$$
\tau(t)=\prod_{k=1}^{n} t_{k}^{1 / p_{k}^{\prime}}, \quad t \in \mathbf{R}_{+}^{n} .
$$

Assume that there is a point $t \in \mathbf{R}_{+}^{n}$ such that $t_{j}>\tau(t)$ for all $j=1, \ldots, n$. Then, applying (4.2), we obtain

$$
\tau(t)=\prod_{j=1}^{n} t_{j}^{1 / p_{j}^{\prime}}>\tau(t)^{1 / p_{1}^{\prime}+\ldots+1 / p_{n}^{\prime}}=\tau(t),
$$

which is false.

Let $f \in \mathscr{M}_{\mathrm{dec}}\left(\mathbf{R}_{+}^{n}\right)$. Observe that

$$
f(t) \leq\left\|f\left(\hat{t}_{j}, \cdot\right)\right\|_{\infty} \equiv \psi_{j}\left(\hat{t}_{j}\right), \quad t \in \mathbf{R}_{+}^{n}, \quad j=1, \ldots, n .
$$


Set

$$
\tau_{j}\left(\hat{t}_{j}\right)=\left(\prod_{k \neq j} t_{k}^{1 / p_{k}^{\prime}}\right)^{p_{j}}
$$

Then

$$
A_{j}=\left\{t \in \mathbf{R}_{+}^{n}: \hat{t}_{j} \in \mathbf{R}_{+}^{n-1}, 0 \leq t_{j} \leq \tau_{j}\left(\hat{t}_{j}\right)\right\} .
$$

Applying (4.5), we have

$$
\begin{aligned}
\int_{A_{j}} \prod_{k=1}^{n} t_{k}^{1 / p_{k}-1} f(t) d t & \leq \int_{\mathbf{R}_{+}^{n-1}} \prod_{k \neq j} t_{k}^{1 / p_{k}-1} \psi_{j}\left(\hat{t}_{j}\right) \int_{0}^{\tau_{j}\left(\hat{t}_{j}\right)} t_{j}^{1 / p_{j}-1} d t_{j} d \hat{t}_{j} \\
& =p_{j} \int_{\mathbf{R}_{+}^{n-1}} \psi_{j}\left(\hat{t}_{j}\right) d \hat{t}_{j}=p_{j}\|f\|_{\mathscr{V}_{j}} .
\end{aligned}
$$

From here and (4.4),

$$
\int_{\mathbf{R}_{+}^{n}} \prod_{k=1}^{n} t_{k}^{1 / p_{k}-1} f(t) d t \leq \sum_{j=1}^{n} p_{j}\|f\|_{\mathscr{V}_{j}} .
$$

Now we derive the multiplicative inequality (4.3) from (4.6). For $\varepsilon_{1}, \ldots, \varepsilon_{n}>0$, set

$$
\varepsilon=\prod_{k=1}^{n} \varepsilon_{k} \quad \text { and } \quad g(t)=f\left(\varepsilon_{1} t_{1}, \ldots, \varepsilon_{n} t_{n}\right), \quad t \in \mathbf{R}_{+}^{n} .
$$

Then $g \in \mathscr{M}_{\mathrm{dec}}\left(\mathbf{R}_{+}^{n}\right)$. Furthermore,

$$
\|g\|_{\mathscr{V} k}=\frac{\varepsilon_{k}}{\varepsilon}\|f\|_{\mathscr{V} k} \quad(k=1, \ldots, n)
$$

and

$$
\int_{\mathbf{R}_{+}^{n}} \prod_{k=1}^{n} t_{k}^{1 / p_{k}-1} g(t) d t=\prod_{k=1}^{n} \varepsilon_{k}^{-1 / p_{k}} \int_{\mathbf{R}_{+}^{n}} \prod_{k=1}^{n} t_{k}^{1 / p_{k}-1} f(t) d t .
$$

Applying (4.6) to the function $g$, we have

$$
\int_{\mathbf{R}_{+}^{n}} \prod_{k=1}^{n} t_{k}^{1 / p_{k}-1} g(t) d t \leq \sum_{k=1}^{n} p_{k}\|g\|_{\mathscr{V}_{k}} .
$$

Further, using (4.7) and (4.8), we get

$$
\int_{\mathbf{R}_{+}^{n}} \prod_{k=1}^{n} t_{k}^{1 / p_{k}-1} f(t) d t \leq \prod_{k=1}^{n} \varepsilon_{k}^{-1 / p_{k}^{\prime}} \sum_{k=1}^{n} p_{k} \varepsilon_{k}\|f\|_{\mathscr{Y}_{k}} .
$$

Now set $\varepsilon_{k}=\left(p_{k}^{\prime} p_{k}\|f\|_{\mathscr{V}_{k}}\right)^{-1}, k=1, \ldots, n$. Using (4.9) and taking into account (4.2), we obtain (4.3).

Corollary 4.3. Let $n \geq 2$ and $1<p<(n-1)^{\prime}$. Set $r=p^{\prime} /(n-1)$. Assume that $f \in \mathscr{V}\left(\mathbf{R}^{n}\right)$. Then

$$
\int_{\mathbf{R}_{+}^{n}} \prod_{k=1}^{n-1} t_{k}^{1 / p-1} t_{n}^{1 / r-1} \mathscr{R}_{\sigma} f(t) d t \leq\left(r r^{\prime}\|f\|_{\mathscr{V}_{n}}\right)^{1 / r^{\prime}} \prod_{k=1}^{n-1}\left(p p^{\prime}\|f\|_{\mathscr{V}_{k}}\right)^{1 / p^{\prime}}
$$


for any $\sigma \in \mathscr{P}_{n}$. In particular, $f \in \mathscr{L}_{\sigma}^{n^{\prime}, 1}\left(\mathbf{R}^{n}\right)$ and

$$
\|f\|_{\mathscr{L}_{\sigma}^{n^{\prime}, 1}} \leq n n^{\prime} \prod_{k=1}^{n}\|f\|_{\mathscr{V}_{k}}^{1 / n} .
$$

for any $\sigma \in \mathscr{P}_{n}$.

Indeed, setting $p_{1}=\cdots=p_{n-1}=p$ and $p_{n}=r$, we have that

$$
\sum_{k=1}^{n} \frac{1}{p_{k}^{\prime}}=\frac{n-1}{p^{\prime}}+\frac{1}{r^{\prime}}=\frac{1}{r}+\frac{1}{r^{\prime}}=1 .
$$

Thus, the condition (4.2) in Theorem 4.2 is satisfied. Applying this theorem, we get (4.10). Taking $p=n^{\prime}$ in (4.10), we obtain (4.11). By (2.4), $\|f\|_{L^{n^{\prime}, 1}} \leq\|f\|_{\mathscr{L}_{\sigma}^{n^{\prime}, 1}}$. Thus, estimate (4.11) implies Theorem 1.3 (although with a worse constant coefficient). We emphasize that the norm in $\mathscr{L}_{\sigma}^{n^{\prime}, 1}\left(\mathbf{R}^{n}\right)$ is stronger than the norm in $L^{n^{\prime}, 1}\left(\mathbf{R}^{n}\right)$, and therefore (4.11) gives a refinement of Theorem 1.3.

Applying Theorem 4.2 and (1.7), we obtain the following embedding for Sobolev spaces.

Corollary 4.4. Let $n \geq 2,1 \leq p_{1}, \ldots, p_{n}<\infty$, and $\sum_{k=1}^{n} 1 / p_{k}^{\prime}=1$. If $f \in$ $W_{1}^{1}\left(\mathbf{R}^{n}\right)$, then

$$
\int_{\mathbf{R}_{+}^{n}} \prod_{k=1}^{n} t_{k}^{1 / p_{k}-1} \mathscr{R}_{\sigma} f(t) d t \leq \prod_{k=1}^{n}\left(p_{k} p_{k}^{\prime}\left\|D_{k} f\right\|_{1}\right)^{1 / p_{k}^{\prime}}
$$

for all $\sigma \in \mathscr{P}_{n}$.

For $p_{1}=\cdots=p_{n}=n^{\prime}$ inequality (4.12) becomes

$$
\|f\|_{\mathscr{L}_{\sigma}^{n^{\prime}, 1}} \leq n n^{\prime} \prod_{k=1}^{n}\left\|D_{k} f\right\|_{1}^{1 / n} .
$$

This estimate coincides with (4.1) (for $p=1$ ).

\section{Mixed norm spaces $\mathscr{V}^{p}$}

In this section we introduce the scale of "intermediate" mixed norm spaces $\mathscr{V}^{p}\left(\mathbf{R}^{n}\right)$. First we recall some definitions. For a function $f \in S_{0}\left(\mathbf{R}^{n}\right)$, set

$$
f^{* *}(t)=\frac{1}{t} \int_{0}^{t} f^{*}(u) d u \text {. }
$$

It is clear that

$$
\lim _{t \rightarrow 0+} f^{* *}(t)=\|f\|_{L^{\infty}} .
$$

For $0<p<\infty$, the space $L^{\infty, p}\left(\mathbf{R}^{n}\right)$ consists of all $f \in S_{0}\left(\mathbf{R}^{n}\right)$ such that

$$
\|f\|_{\infty, p} \equiv\left(\int_{0}^{\infty}\left[f^{* *}(t)-f^{*}(t)\right]^{p} \frac{d t}{t}\right)^{1 / p}<\infty
$$

(see [1], [13]). Applying the equality

$$
\frac{d}{d t} f^{* *}(t)=-\frac{1}{t}\left(f^{* *}(t)-f^{*}(t)\right) \quad(t>0)
$$


and using (5.1), we obtain that $L^{\infty, 1}\left(\mathbf{R}^{n}\right)=L^{\infty}\left(\mathbf{R}^{n}\right)$ and

$$
\|f\|_{\infty, 1}=\|f\|_{\infty} \quad \text { for any } f \in S_{0}\left(\mathbf{R}^{n}\right) \text {. }
$$

Further, let $1<r<\infty$. Assume that $f \in L^{r, 1}\left(\mathbf{R}^{n}\right)$. Applying Fubini's theorem, we have

This implies that

$$
\int_{0}^{\infty} t^{1 / r} f^{*}(t) \frac{d t}{t}=\frac{1}{r^{\prime}} \int_{0}^{\infty} t^{1 / r} f^{* *}(t) \frac{d t}{t}
$$

$$
\frac{1}{r^{\prime}} \int_{0}^{\infty} t^{1 / r}\left[f^{* *}(t)-f^{*}(t)\right] \frac{d t}{t}=\frac{1}{r}\|f\|_{r, 1} .
$$

Applying (5.2), (5.3), and the monotone convergence theorem (for both increasing and decreasing sequences), we easily obtain that

$$
\lim _{r \rightarrow \infty} \frac{1}{r}\|f\|_{r, 1}=\|f\|_{\infty} \quad \text { if } f \in L^{r_{0}, 1}\left(\mathbf{R}^{n}\right) \text { for some } r_{0}>1 \text {. }
$$

The spaces $\mathscr{V}_{k}$ are defined as mixed norm spaces in which the interior norm is $L^{\infty}$-norm (see (1.5)). That is, for a function $f \in \mathscr{V}_{k}\left(\mathbf{R}^{n}\right)$ almost all linear sections $f_{\hat{x}_{k}}$ are essentially bounded on $\mathbf{R}$. Basing on equalities (5.2) and (5.3), it is natural to impose growth conditions on linear sections of functions in terms of $L^{r, 1}(\mathbf{R})$-norms $(1 \leq r \leq \infty)$. Then Corollary 4.3 suggests that the corresponding scale of exterior norms should be formed by the spaces $L^{p, 1}\left(\mathbf{R}^{n-1}\right)$, where

$$
\frac{1}{r^{\prime}}+\frac{n-1}{p^{\prime}}=1 \text {. }
$$

These reasonings together with observations given in Introduction (see (1.12)) lead us to the following definition.

Let $n \geq 2$ and $1 \leq p \leq(n-1)^{\prime}$. Set $r_{p}=p^{\prime} /(n-1)$. For a function $f \in S_{0}\left(\mathbf{R}^{n}\right)$ and $k=1, \ldots, n$, set

$$
\psi_{k}^{(p)}\left(\hat{x}_{k}\right)=\left\|f\left(\hat{x}_{k}, \cdot\right)\right\|_{L^{r_{p}, 1}(\mathbf{R})} \quad \text { and } \quad\|f\|_{\mathscr{V}_{k}^{p}}=\left\|\psi_{k}^{(p)}\right\|_{L^{p, 1}\left(\mathbf{R}^{n-1}\right)} .
$$

By $\mathscr{V}_{k}^{p}\left(\mathbf{R}^{n}\right)$ we denote the class of all functions $f \in S_{0}\left(\mathbf{R}^{n}\right)$ such that $\|f\|_{\mathscr{V}_{k}^{p}}<\infty$. As usual, we write

Set also

$$
\mathscr{V}_{k}^{p}\left(\mathbf{R}^{n}\right)=L_{\hat{x}_{k}}^{p, 1}\left(\mathbf{R}^{n-1}\right)\left[L_{x_{k}}^{r_{p}, 1}(\mathbf{R})\right]
$$

$$
\mathscr{V}^{p}\left(\mathbf{R}^{n}\right)=\bigcap_{k=1}^{n} \mathscr{V}_{k}^{p}\left(\mathbf{R}^{n}\right), \quad\|f\|_{\mathscr{V} p}=\sum_{k=1}^{n}\|f\|_{\mathscr{V}_{k}^{p}} .
$$

In this section we study embeddings of the space $\mathscr{V}$ into the spaces $\mathscr{V}^{p}$. Observe that $\mathscr{V}_{k}^{1}=\mathscr{V}_{k}$, and the norms coincide. Indeed, if $p=1$, then $r_{p}=\infty$, and by (5.2)

$$
\|f\|_{\mathscr{V}_{k}^{1}\left(\mathbf{R}^{n}\right)}=\int_{\mathbf{R}^{n-1}}\left\|f\left(\hat{x}_{k}, \cdot\right)\right\|_{\infty, 1} d \hat{x}_{k}=\int_{\mathbf{R}^{n-1}}\left\|f\left(\hat{x}_{k}, \cdot\right)\right\|_{\infty} d \hat{x}_{k}=\|f\|_{\mathscr{V}_{k}\left(\mathbf{R}^{n}\right)} .
$$

Further, we note that the case $n=2$ and $p=\infty$ also is included to the definition of $\mathscr{V}_{k}^{p}$. In this case $r=p^{\prime}=\infty$, and by (5.2),

$$
\mathscr{V}_{1}^{\infty}\left(\mathbf{R}^{2}\right)=L_{x_{2}}^{\infty, 1}(\mathbf{R})\left[L_{x_{1}}^{1}(\mathbf{R})\right]=L_{x_{2}}^{\infty}(\mathbf{R})\left[L_{x_{1}}^{1}(\mathbf{R})\right]
$$

and similarly

$$
\mathscr{V}_{2}^{\infty}\left(\mathbf{R}^{2}\right)=L_{x_{1}}^{\infty}(\mathbf{R})\left[L_{x_{2}}^{1}(\mathbf{R})\right]
$$


moreover, the corresponding norms coincide. Further, we have that

$$
\|f\|_{\mathscr{V}_{1}^{\infty}} \leq\|f\|_{\mathscr{V}_{2}} \text { and }\|f\|_{\mathscr{V}_{2}^{\infty}} \leq\|f\|_{\mathscr{V}_{1}} .
$$

Indeed, the first inequality in (5.5) is equivalent to the obvious estimate

$$
\operatorname{ess} \sup _{x_{2} \in \mathbf{R}} \int_{\mathbf{R}}\left|f\left(x_{1}, x_{2}\right)\right| d x_{1} \leq \int_{\mathbf{R}} \operatorname{ess}_{\sup _{x_{2} \in \mathbf{R}}}\left|f\left(x_{1}, x_{2}\right)\right| d x_{1} .
$$

The second inequality in (5.5) is obtained similarly.

Theorem 5.1. Let $n \geq 2,1<p \leq(n-1)^{\prime}$, and $r=p^{\prime} /(n-1)$. If $f \in \mathscr{V}\left(\mathbf{R}^{n}\right)$, then $f \in \mathscr{V}^{p}\left(\mathbf{R}^{n}\right)$, and for every $j=1, \ldots, n$ it holds that

$$
\|f\|_{\mathscr{V}_{j}^{p}} \leq c_{n, p}\|f\|_{\mathscr{V}_{j}}^{1 / r^{\prime}} \prod_{k \neq j}\|f\|_{\mathscr{V}_{k}}^{1 / p^{\prime}}
$$

where $c_{n, p}=1$ if $n=2$ and $p=\infty$, and $c_{n, p}=\left(r r^{\prime}\right)^{1 / r^{\prime}}\left(p p^{\prime}\right)^{(n-1) / p^{\prime}}$ otherwise.

Proof. In the case $n=2$ and $p=\infty$ inequality (5.6) coincides with (5.5). Assume that either $n=2$ and $1<p<\infty$, or $n \geq 3$ and $1<p \leq(n-1)^{\prime}$. We will prove (5.6) for $j=n$.

Let $\mathscr{R}_{n} f(y, \eta)\left(y \in \mathbf{R}^{n-1}, \eta \in \mathbf{R}_{+}\right)$be the rearrangement of $f$ with respect to the $n$th variable. Next, for a fixed $\eta \in \mathbf{R}_{+}$, let $F(\xi, \eta)\left(\xi \in \mathbf{R}_{+}\right)$be the rearrangement of the function

$$
y \mapsto \mathscr{R}_{n} f(y, \eta), \quad y \in \mathbf{R}^{n-1},
$$

with respect to $y$. It follows from [4, Theorem 4.5. I] that

$$
\|f\|_{\mathscr{F}_{n}^{p}} \leq \int_{0}^{\infty} \int_{0}^{\infty} \xi^{1 / p-1} \eta^{1 / r-1} F(\xi, \eta) d \xi d \eta
$$

On the other hand, let $\sigma=(n, n-1, \ldots, 1)$. For a fixed $\eta \in \mathbf{R}_{+}$, we take the iterative rearrangement of the function (5.7) successively with respect to the variables $y_{n-1}, \ldots, y_{1}$. We obtain the rearrangement $\mathscr{R}_{\sigma} f(s, \eta), s \in \mathbf{R}_{+}^{n-1}$. By Theorem 2.1, for any fixed $\eta$, we have

$$
\int_{0}^{\infty} \xi^{1 / p-1} F(\xi, \eta) d \xi \leq \int_{\mathbf{R}_{+}^{n-1}}\left(\prod_{k=1}^{n-1} s_{k}\right)^{1 / p-1} \mathscr{R}_{\sigma} f(s, \eta) d s .
$$

Further, by Corollary 4.3,

$$
\begin{aligned}
& \int_{\mathbf{R}_{+}^{n-1}} \int_{\mathbf{R}_{+}}\left(\prod_{k=1}^{n-1} s_{k}\right)^{1 / p-1} \eta^{1 / r-1} \mathscr{R}_{\sigma} f(s, \eta) d s d \eta \\
& \leq\left(r r^{\prime}\|f\|_{\mathscr{V}_{n}}\right)^{1 / r^{\prime}} \prod_{k=1}^{n-1}\left(p p^{\prime}\|f\|_{\mathscr{V}_{k}}\right)^{1 / p^{\prime}} .
\end{aligned}
$$

Applying inequalities (5.8), (5.9), and (5.10), we obtain (5.6).

Remark 5.2. If $n \geq 3$, then the spaces $\mathscr{V}_{k}^{p}\left(\mathbf{R}^{n}\right)$ formally can be defined for $p>(n-1)^{\prime}$, too (with $r_{p}=p^{\prime} /(n-1)<1$ ). However, in this case Theorem 5.1 fails to hold. Indeed, take $(n-1) / p<\alpha<n-2$ and set

$$
f(x)=\sum_{k=1}^{n}\left|\hat{x}_{j}\right|^{-\alpha} \chi_{(0,1)^{n}}(x), \quad x \in \mathbf{R}^{n},
$$


A straightforward computation shows that $f \in \mathscr{V}\left(\mathbf{R}^{n}\right)$ and for any $r>0$

$$
\|f\|_{L_{\hat{x}_{j}}^{p}\left(\mathbf{R}^{n-1}\right)\left[L_{x_{j}}^{r}(\mathbf{R})\right]}=\infty \quad(j=1, \ldots, n) .
$$

Applying (5.6) and the theorem on the arithmetic and geometric means, we easily obtain the following:

Corollary 5.3. Let either $n=2$ and $1<p<\infty$, or $n \geq 3$ and $1<p \leq(n-1)^{\prime}$. Set $r \equiv r_{p}=p^{\prime} /(n-1)$. If $f \in \mathscr{V}\left(\mathbf{R}^{n}\right)$, then $f \in \mathscr{V}^{p}\left(\mathbf{R}^{n}\right)$ and

$$
\|f\|_{\mathscr{V}_{k}^{p}} \leq r_{p}\|f\|_{\mathscr{V}_{k}}+p \sum_{j \neq k}\|f\|_{\mathscr{V}_{j}} \quad(k=1, \ldots, n) .
$$

We shall show below that (5.11) (divided by $r_{p}$ ) becomes equality as $p \rightarrow 1$.

Remark 5.4. Let $n=2$ and $f \in \mathscr{V}\left(\mathbf{R}^{2}\right)$. By (5.6), we have that

$$
\|f\|_{\mathscr{V}_{1}^{p}} \leq p p^{\prime}\|f\|_{\mathscr{V}_{1}}^{1 / p}\|f\|_{\mathscr{V}_{2}}^{1 / p^{\prime}} \quad(1<p<\infty)
$$

and

$$
\|f\|_{\mathscr{V}_{1}^{\infty}} \leq\|f\|_{\mathscr{V}_{2}}
$$

Observe that for $f=\chi_{(0,1)^{2}}$ we have equalities in (5.12) and (5.13). Hence, the constants in these inequalities are optimal. However, we notice that the constant in (5.12) tends to $\infty$ as $p \rightarrow \infty$, but for $p=\infty$ we have inequality (5.13) with the constant 1 . It is easy to explain this fact. Indeed, it follows directly from (5.12) that

$$
\limsup _{p \rightarrow \infty} \frac{1}{p}\|f\|_{\mathscr{V}_{1}^{p}} \leq\|f\|_{\mathscr{V}_{2}}
$$

Besides, we show below that

$$
\|f\|_{\mathscr{V}_{1}^{\infty}} \leq \liminf _{p \rightarrow \infty} \frac{1}{p}\|f\|_{\mathscr{V}_{1}^{p}} .
$$

By virtue of relations (5.14) and (5.15), (5.13) follows from (5.12) as a limiting case as $p \rightarrow \infty$.

To prove (5.15), we observe that for any $y \in \mathbf{R}$ and any $\mu>0$,

$$
\|f(\cdot, y)\|_{p^{\prime}, 1} \geq \int_{0}^{\mu} s^{1 / p^{\prime}-1} \mathscr{R}_{1} f(s, y) d s \geq \mu^{1 / p^{\prime}-1} F_{\mu}(y),
$$

where

Thus,

$$
F_{\mu}(y)=\int_{0}^{\mu} \mathscr{R}_{1} f(s, y) d s, \quad y \in \mathbf{R}
$$

$$
\|f\|_{\mathscr{V}_{1}^{p}} \geq \mu^{1 / p^{\prime}-1}\left\|F_{\mu}\right\|_{p, 1}
$$

On the other hand,

for any $\tau>0$, and therefore

$$
\left\|F_{\mu}\right\|_{p, 1} \geq p \tau^{1 / p} F_{\mu}^{*}(\tau)
$$

$$
\liminf _{p \rightarrow \infty} \frac{1}{p}\|f\|_{\mathscr{V}_{1}^{p}} \geq\left\|F_{\mu}\right\|_{\infty}
$$

(by virtue of (5.16), it follows also from (5.4)). It is easy to see that $\|f\|_{\mathscr{V}_{1}^{\infty}}=$ $\lim _{\mu \rightarrow \infty}\left\|F_{\mu}\right\|_{\infty}$. Thus, we obtain (5.15).

Inequalities (5.11) and (1.7) yield the following: 
Corollary 5.5. If $f \in W_{1}^{1}\left(\mathbf{R}^{n}\right)(n \geq 2)$, then $f \in \mathscr{V}^{p}\left(\mathbf{R}^{n}\right)$ for any $1<p \leq$ $(n-1)^{\prime}$, and

$$
\|f\|_{\mathscr{V}^{p}} \leq c \sum_{k=1}^{n}\left\|D_{k} f\right\|_{1},
$$

where $c$ depends only on $p$ and $n$.

Remark 5.6. The results of this section have been derived from the iterative rearrangement inequality (4.3) (see the proof of Theorem 5.1). However, these results are expressed in terms of mixed norm spaces and therefore they give a more explicit description of the behaviour of linear sections of functions. In particular, taking $p=n^{\prime}$ in Corollary 5.5, we obtain:

$$
W_{1}^{1}\left(\mathbf{R}^{n}\right) \subset L_{\hat{x}_{k}}^{n^{\prime}, 1}\left(\mathbf{R}^{n-1}\right)\left[L_{x_{k}}^{n^{\prime}, 1}(\mathbf{R})\right], \quad k=1, \ldots, n .
$$

For $n=2$ we have that

$$
W_{1}^{1}\left(\mathbf{R}^{2}\right) \subset L_{x_{2}}^{2,1}(\mathbf{R})\left[L_{x_{1}}^{2,1}(\mathbf{R})\right] \cap L_{x_{1}}^{2,1}(\mathbf{R})\left[L_{x_{2}}^{2,1}(\mathbf{R})\right] .
$$

This inclusion does not follow from the strong type Sobolev inequality (1.8), which states that

$$
W_{1}^{1}\left(\mathbf{R}^{2}\right) \subset L^{2,1}\left(\mathbf{R}^{2}\right) .
$$

Indeed, it was shown by Cwikel [5] that

$$
L^{2,1}\left(\mathbf{R}^{2}\right) \not \subset L_{x_{2}}^{2,1}(\mathbf{R})\left[L_{x_{1}}^{2,1}(\mathbf{R})\right] .
$$

At the same time, the results in terms of iterative rearrangements are stronger. In particular, if $f \in W_{1}^{1}\left(\mathbf{R}^{2}\right)$, then $f \in \mathscr{L}^{2,1}\left(\mathbf{R}^{2}\right)$ (see Theorem 4.1). Mixed norm inequalities follow from here, since

$$
\mathscr{L}_{\{1,2\}}^{2,1}\left(\mathbf{R}^{2}\right) \subset L_{x_{2}}^{2,1}(\mathbf{R})\left[L_{x_{1}}^{2,1}(\mathbf{R})\right], \mathscr{L}_{\{2,1\}}^{2,1}\left(\mathbf{R}^{2}\right) \subset L_{x_{1}}^{2,1}(\mathbf{R})\left[L_{x_{2}}^{2,1}(\mathbf{R})\right]
$$

(see [4, Theorem 4.5. I]).

Finally, we return to inequality (5.11) and we shall study the limiting behaviour of $\|f\|_{\mathscr{Y}_{j}^{p}}$ as $p \rightarrow 1+$. We observe that for any $s \geq 1$

$$
\frac{1}{s}\|f\|_{s, 1}=\frac{1}{s} \int_{0}^{\infty} t^{1 / s-1} f^{*}(t) d t=\int_{0}^{\infty} f^{*}\left(u^{s}\right) d u
$$

(note that (5.4) can be also easily derived from (5.17)).

Theorem 5.7. Let $n \geq 2$ and let $k \in\{1, \ldots, n\}$. If $f \in S_{0}\left(\mathbf{R}^{n}\right)$ and

$$
f \in \bigcap_{j \neq k} \mathscr{V}_{j}\left(\mathbf{R}^{n}\right)
$$

then

$$
\lim _{p \rightarrow 1+} \frac{n-1}{p^{\prime}}\|f\|_{\mathscr{V}_{k}^{p}}=\|f\|_{\mathscr{V}_{k}}
$$

Proof. We prove (5.19) for $k=n$. Let $\left\{p_{\nu}\right\}$ be a decreasing sequence of numbers such that $p_{\nu}>1$ and $p_{\nu} \rightarrow 1$. Let $s_{\nu}=p_{\nu}^{\prime} /(n-1)$. Then $\left\{s_{\nu}\right\}$ increases and $s_{\nu} \rightarrow \infty$. Set

$$
F_{\nu}(y)=\frac{1}{s_{\nu}}\|f(y, \cdot)\|_{L^{s_{\nu}, 1}(\mathbf{R})}, \quad y \in \mathbf{R}^{n-1} .
$$


By (5.17),

Set also

$$
F_{\nu}(y)=\int_{0}^{\infty} \mathscr{R}_{n} f\left(y, u^{s_{\nu}}\right) d u
$$

$$
G_{\nu}(y)=\int_{0}^{1} \mathscr{R}_{n} f\left(y, u^{s_{\nu}}\right) d u .
$$

For a fixed $y \in \mathbf{R}^{n-1}$, the functions $u \mapsto \mathscr{R}_{n} f\left(y, u^{s_{\nu}}\right)$ form an increasing sequence on $(0,1)$ and

$$
\lim _{\nu \rightarrow \infty} \mathscr{R}_{n} f\left(y, u^{s_{\nu}}\right)=\mathscr{R}_{n} f(y, 0+)=\|f(y, \cdot)\|_{L^{\infty}(\mathbf{R})}, \quad u \in(0,1) .
$$

Setting $\|f(y, \cdot)\|_{L^{\infty}(\mathbf{R})}=\varphi(y)$, and applying the monotone convergence theorem, we obtain that

$$
\lim _{\nu \rightarrow \infty} G_{\nu}(y)=\varphi(y) \quad \text { for any } y \in \mathbf{R}^{n-1} .
$$

Moreover, $\left\{G_{\nu}(y)\right\}$ is an increasing sequence on $\mathbf{R}^{n-1}$ and thus

$$
\lim _{\nu \rightarrow \infty} G_{\nu}^{*}(t)=\varphi^{*}(t) \quad \text { for any } t>0
$$

(see [2, p. 41]).

We shall prove that

$$
\lim _{\nu \rightarrow \infty} \frac{1}{s_{\nu}}\|f\|_{\mathscr{V}_{n}^{p_{\nu}}}=\|f\|_{\mathscr{V}_{n}}
$$

We have $F_{\nu}^{*}(t) \geq G_{\nu}^{*}(t)$ and therefore

$$
\frac{1}{s_{\nu}}\|f\|_{\mathscr{V}_{n}^{p_{\nu}}}=\int_{0}^{\infty} t^{1 / p_{\nu}-1} F_{\nu}^{*}(t) d t \geq \int_{0}^{\infty} t^{1 / p_{\nu}-1} G_{\nu}^{*}(t) d t .
$$

For any fixed $t \in(1, \infty)$ the sequence $\left\{t^{1 / p_{\nu}-1} G_{\nu}^{*}(t)\right\}$ increases. Thus, by (5.20) and the monotone convergence theorem, we have that

$$
\lim _{\nu \rightarrow \infty} \int_{1}^{\infty} t^{1 / p_{\nu}-1} G_{\nu}^{*}(t) d t=\int_{1}^{\infty} \varphi^{*}(t) d t .
$$

The monotone convergence theorem implies also that

$$
\lim _{\nu \rightarrow \infty} \int_{0}^{1} G_{\nu}^{*}(t) d t=\int_{0}^{1} \varphi^{*}(t) d t .
$$

Now, applying (5.22), (5.23), and (5.24), we obtain that

$$
\|f\|_{\mathscr{V}_{n}}=\|\varphi\|_{1} \leq \liminf _{\nu \rightarrow \infty} \int_{0}^{\infty} t^{1 / p_{\nu}-1} G_{\nu}^{*}(t) d t \leq \liminf _{\nu \rightarrow \infty} \frac{1}{s_{\nu}}\|f\|_{\mathscr{V}_{n}^{p_{\nu}}}
$$

First, this implies (5.21) if $\|f\|_{\mathscr{V}_{n}}=\infty$. Suppose now that $\|f\|_{\mathscr{V}_{n}}<\infty$. Then, by virtue of assumption (5.18), we have that $f \in \mathscr{V}\left(\mathbf{R}^{n}\right)$. Thus, by inequality (5.11),

$$
\frac{1}{s_{\nu}}\|f\|_{\mathscr{V}_{n}^{p}} \leq\|f\|_{\mathscr{V}_{n}}+\frac{p_{\nu}}{s_{\nu}} \sum_{j \neq k}\|f\|_{\mathscr{V}_{j}} .
$$

Since $p_{\nu} / s_{\nu} \rightarrow 0$ as $\nu \rightarrow \infty$, it follows that

$$
\limsup _{\nu \rightarrow \infty} \frac{1}{s_{\nu}}\|f\|_{\mathscr{Y}_{n}^{p_{\nu}}} \leq\|f\|_{\mathscr{V}_{n}}
$$

Inequalities (5.25) and (5.26) combined give (5.21). This proves the theorem. 
Corollary 5.8. If $f \in S_{0}\left(\mathbf{R}^{n}\right)(n \geq 2)$, then

$$
\lim _{p \rightarrow 1+} \frac{n-1}{p^{\prime}}\|f\|_{\mathscr{V} p}=\|f\|_{\mathscr{V}}
$$

\section{References}

[1] Bastero, J., M. Milman, and F. J. Ruiz Blasco: A note on $L(\infty, q)$ spaces and Sobolev embeddings. - Indiana Univ. Math. J. 52:5, 2003, 1215-1230.

[2] Bennett, C., and R. Sharpley: Interpolation of operators. - Pure and Applied Mathematics 129, Academic Press, Boston, MA, 1988.

[3] Blei, R. C., and J. J. F. Fournier: Mixed-norm conditions and Lorentz norms. - In: Commutative harmonic analysis (Canton, NY, 1987), Contemp. Math. 91, Amer. Math. Soc., Providence, RI, 1989, 57-78.

[4] Blozinski, A. P.: Multivariate rearrangements and Banach function spaces with mixed norms. - Trans. Amer. Math. Soc. 263:1, 1981, 149-167.

[5] Cwikel, M.: On $\left(L^{p o}\left(A_{o}\right), L^{p_{1}}\left(A_{1}\right)\right)_{\theta}, q$. - Proc. Amer. Math. Soc. 44, 1974, 286-292.

[6] Fournier, J.: Mixed norms and rearrangements: Sobolev's inequality and Littlewood's inequality. - Ann. Mat. Pura Appl. 148:4, 1987, 51-76.

[7] Gagliardo, E.: Proprietà di alcune classi di funzioni in più variabili. - Ricerche Mat. 7, 1958, $102-137$.

[8] KolyadA, V. I.: Embeddings of fractional Sobolev spaces and estimates of Fourier transforms. - Mat. Sb. 192:7, 2001, 51-72 (in Russian); English transl. in Sb. Math. 192:7-8, 2001, 979-1000.

[9] Kolyada, V. I.: Mixed norms and Sobolev type inequalities. - In: Approximation and probability, Banach Center Publ. 72, Polish Acad. Sci., Warsaw, 2006, 141-160.

[10] Kolyada, V. I.: On embedding theorems. - In: Nonlinear Analysis, Function Spaces and Applications 8 (Proceedings of the Spring School held in Prague, 2006), Prague, 2007, 35-94.

[11] Loomis, L. H., and H. Whitney: An inequality related to the isoperimetric inequality. - Bull. Amer. Math. Soc. 55, 1949, 961-962.

[12] Lorentz, G. G.: On the theory of spaces $\Lambda$. - Pacific J. Math. 1, 1951, 411-429.

[13] Malý, J., and L. Pick: An elementary proof of sharp Sobolev embeddings. - Proc. Amer. Math. Soc. 130:2, 2001, 555-563.

[14] Milman, M.: Notes on interpolation of mixed norm spaces and applications. - Quart. J. Math. Oxford Ser. (2) 42:167, 1991, 325-334.

[15] O'NeIL, R.: Convolution operators and $L(p, q)$ spaces. - Duke Math. J. 30, 1963, 129-142.

[16] Peetre, J.: Espaces d'interpolation et espaces de Soboleff. - Ann. Inst. Fourier (Grenoble) $16,1966,279-317$.

[17] Poornima, S.: An embedding theorem for the Sobolev space $W^{1,1}$. - Bull. Sci. Math. 107:2, 1983, 253-259.

[18] Yatsenko, A. A.: Iterative rearrangements of functions, and Lorentz spaces. - Izv. Vyssh. Uchebn. Zaved. Mat. 5, 1998, 73-77 (in Russian); English transl. in Russian Math. (Iz. VUZ) $42: 5,1998,71-75$. 